\title{
Utilidad del complejo soluble de ataque a membrana para determinar actividad clínica de la dermatomiositis y respuesta al tratamiento
}

\author{
Andrés Tirado-Sánchez, Alexandro Bonifaz y Rosa María Ponce-Olivera \\ Secretaría de Salud, Hospital General de México, Servicio de Dermatología, Ciudad de México, México
}

\begin{abstract}
Resumen
Introducción: La dermatomiositis es una enfermedad autoinmune y es la más común de las miopatías inflamatorias idiopáticas. Durante el seguimiento de los pacientes se requiere determinar parámetros bioquímicos para precisar la actividad de la enfermedad y la eficacia de los tratamientos. Objetivo: Definir la relación entre el grado de activación del sistema del complemento a través del complejo soluble de ataque a membrana (C5b-9), la actividad clínica de la dermatomiositis y sus variaciones con el tratamiento convencional. Método: Se estudiaron 45 pacientes con dermatomiositis activa e inactiva. Se establecieron parámetros bioquímicos, severidad clínica y se correlacionaron con los niveles séricos de C5b-9, determinados mediante ELISA. Resultados: Existió correlación positiva entre la actividad cutánea y muscular de la dermatomiositis y los niveles séricos de C5b-9, menor que con los marcadores bioquímicos tradicionales. En la respuesta al tratamiento, C5b-9 mostró reducción significativa, similar a la severidad clínica; con los parámetros bioquímicos, la reducción no fue significativa a un mes de tratamiento con esteroides sistémicos. Conclusiones: Los niveles séricos de C5b-9 en pacientes con dermatomiositis están más elevados que en los sujetos sanos; con su medición se identificaron los casos activos e inactivos de dermatomiositis. Pueden ser un parámetro fiable de respuesta terapéutica, más precisos que la medición de enzimas musculares, particularmente creatinfosfosquinasa.
\end{abstract}

PALABRAS CLAVE: Dermatomiositis. Actividad. Sistema del complemento. C5b-9. Creatinfosfoquinasa.

\begin{abstract}
Introduction: Dermatomyositis is an autoimmune disease and the most common idiopathic inflammatory myopathy. During patient follow-up, determining biochemical parameters is required in order to assess for disease activity and treatment efficacy. Objective: To determine the relationship between the degree of activation of the complement system through the soluble membrane attack complex (c5b-9), dermatomyositis clinical activity and its variations with conventional treatment. Method: Forty-five patients with active and inactive dermatomyositis were studied. Chemical parameters and clinical severity were established and correlated with ELISA-determined C5b-9 serum levels. Results: There was positive correlation between dermatomyositis cutaneous and muscular activity and C5b-9 serum levels, which was lower than with traditional biochemical markers. In the case of treatment response, C5b-9 showed significant reduction, similar to clinical severity; with biochemical parameters, the reduction was not significant at one month of treatment with systemic steroids. Conclusions: Serum levels of C5b-9 levels of C5b-9 are higher in patients with dermatomyositis than in healthy subjects; dermatomyositis active and inactive cases were determined by means of their measurement. They can be a reliable parameter of therapeutic response, more accurate than muscle enzymes measurement, particularly creatine phosphokinase.
\end{abstract}

KEY WORDS: Dermatomyositis. Activity. Complement system. C5b-9. Creatine phosphokinase.

Correspondencia:

Andrés Tirado-Sánchez

E-mail: atsdermahgm @ gmail.com
Fecha de recepción: 09-08-2017

Fecha de aceptación: 27-07-2018

DOI: 10.24875/GMM.18003637
Gac Med Mex. 2018;154:582-587

Disponible en PubMed

www.gacetamedicademexico.com 


\section{Introducción}

La dermatomiositis es una enfermedad autoinmune incluida en el grupo de las miopatías inflamatorias idiopáticas. ${ }^{1}$ Es una enfermedad con frecuentes complicaciones derivadas de la afectación visceral o de la progresión o reagudización del proceso. ${ }^{2}$ En las últimas décadas se ha intentado identificar marcadores serológicos que faciliten el diagnóstico de la dermatomiositis, ${ }^{3}$ y que además evalúen la respuesta clínica con los diferentes esquemas de tratamiento y en consecuencia permitan realizar un pronóstico de la enfermedad.

Los parámetros utilizados para el diagnóstico (fuerza muscular, enzimas musculares, hallazgos histológicos y electromiografía), aunque son de utilidad no son lo suficientemente sensibles ni específicos para evaluar la respuesta al tratamiento, ${ }^{3}$ por lo que se han estudiado otras opciones relacionadas con la patogenia de la enfermedad y con ello establecer la correlación clínica (por ejemplo, asociados con la lesión muscular, la lesión endotelial, el fenómeno inmunológico, citoquinas, moléculas de adhesión, entre otros), sin embargo, ninguno ha obtenido un grado de correlación suficiente para sustituir a los marcadores bioquímicos y electrofisiológicos tradicionalmente utilizados., ${ }^{2,3}$

Se ha observado que el daño muscular en la dermatomiositis se relaciona con una microangiopatía mediada por el complemento, ${ }^{4,5}$ describiéndose depósitos del complejo de ataque de membrana o C5b-9 en la microvasculatura muscular de pacientes con la enfermedad, ${ }^{5}$ en diferentes grados de severidad con un gradiente positivo, traduciendo su potencial utilidad en la determinación del grado de actividad de la enfermedad.

El C5b-9 es un complejo de proteínas que se unen unas con otras para formar poros a través de la membrana, resultando en la muerte celular. ${ }^{6-8}$ Varios estudios han mostrado depósitos de este complejo proteico en la piel, fibras musculares y en arteriolas y capilares en biopsias de pacientes con dermatomiositis. ${ }^{9-11}$ La medición de los niveles de productos de activación del complemento es una medida del estado catabólico y, en consecuencia, es más precisa para establecer el grado de activación del mismo.

Hasta el momento existen pocos estudios centrados en el análisis en pacientes con dermatomiositis, por tal motivo realizamos una investigación para determinar la relación entre el grado de activación del sistema del complemento a través del complejo soluble C5b-9, la actividad clínica de la dermatomiositis y sus variaciones con el tratamiento.

\section{Métodos}

El estudio se realizó entre 2005 y 2011 y fue aprobado por el Comité de Ética en Investigación con el registro DI-06-109-3-61. Todos los pacientes y controles sanos dieron su consentimiento para participar en el estudio. Se incluyeron 45 pacientes del sexo femenino entre los 28 y 46 años de edad que cumplieron los criterios de Bohan y Peter: ${ }^{12,13}$

- Grupo 1: pacientes con diagnóstico reciente, no tratados y con enfermedad activa.

- Grupo 2: pacientes con enfermedad inactiva, con o sin tratamiento de mantenimiento.

Se incluyó a un grupo de sujetos sanos pareados por edad con los dos grupos anteriores en proporción 1:1.

Los pacientes fueron valorados al momento de su inclusión al estudio, siempre por el mismo investigador. Se determinó la severidad de la enfermedad utilizando los datos obtenidos de los expedientes clínicos, por interrogatorio y exploración física mediante el uso de la Escala de Evaluación de la Actividad de la Enfermedad (MYOACT), establecida por el Grupo Internacional de Evaluación y Estudios Clínicos de la Miositis. ${ }^{14}$ La puntuación de MYOACT se registró como muscular, cutánea y total. Se excluyeron los pacientes con síndrome de sobreposición o variedades de dermatomiositis diferentes al tipo 2.

Los pacientes considerados con enfermedad inactiva debían mantener una puntuación de 0 en el MYOACT (muscular, cutáneo y total) al menos un mes. Los pacientes que no cumplieran con este criterio eran considerados con enfermedad activa.

Los datos clínicos fueron obtenidos cuando el paciente aceptó participar en el estudio e incluían la edad, el tiempo de evolución de la enfermedad y el MYOACT en sus tres modalidades (cutánea, muscular y total). Se obtuvieron muestras de sangre $(10 \mathrm{~mL})$ para la medición de creatinfosfoquinasa como marcador bioquímico de la dermatomiositis y de C5b-9 sérico. Para los controles, se obtuvieron muestras de sangre de 45 mujeres sanas, pareadas por edad (26 a 45 años), provenientes de banco de sangre del hospital. La muestra de sangre obtenida se utilizó para determinar creatinfosfoquinasa y la concentración sérica de C5b-9 mediante técnica de ELISA, utilizando un kit comercial de C5b-9 sérico humano (MyBiosource, San Diego, CA, EE. UU). Todas las muestras, tanto de casos como controles, fueron analizadas 
Tabla 1. Características clínicas de pacientes con dermatomiositis

\begin{tabular}{|c|c|c|c|c|c|}
\hline & \multicolumn{2}{|c|}{$\begin{array}{l}\text { Enfermedad } \\
\text { activa } \\
(n=28)\end{array}$} & \multicolumn{2}{|c|}{$\begin{array}{l}\text { Enfermedad } \\
\text { inactiva } \\
(n=17)\end{array}$} & \multirow[t]{2}{*}{$\mathbf{P}$} \\
\hline & $\mathbf{n}$ & $\%$ & $\mathbf{n}$ & $\%$ & \\
\hline \multicolumn{6}{|l|}{ Características clínicas } \\
\hline Enfermedad pulmonar intersticial & 5 & 18 & 0 & 0 & 0.001 \\
\hline Disfagia & 18 & 64 & 0 & 0 & 0.001 \\
\hline Fenómeno de Raynaud & 8 & 28 & 0 & 0 & 0.001 \\
\hline Mano de mecánico & 12 & 43 & 0 & 0 & 0.001 \\
\hline \multirow[t]{2}{*}{ Artritis } & 6 & 21 & 0 & 0 & 0.001 \\
\hline & \multicolumn{2}{|c|}{ Media \pm DE } & \multicolumn{2}{|c|}{ Media \pm DE } & \\
\hline \multicolumn{6}{|l|}{ Datos demográficos } \\
\hline Edad de inicio (años) & \multicolumn{2}{|c|}{$\begin{array}{c}34.16 \pm 15.23 \\
(21-66)\end{array}$} & \multicolumn{2}{|c|}{$\begin{array}{c}32.33 \pm 14.55 \\
(19-65)\end{array}$} & ns \\
\hline Duración de los síntomas hasta el momento del estudio (meses) & \multicolumn{2}{|c|}{$\begin{array}{c}8.12 \pm 6.56 \\
(2-17)\end{array}$} & \multicolumn{2}{|c|}{$\begin{array}{c}12.55 \pm 8.85 \\
(4-16)\end{array}$} & ns \\
\hline Niveles de creatinfosfoquinasa al momento de que fue medido el C5b-9 sérico & \multicolumn{2}{|c|}{$1675 \pm 1375$} & \multicolumn{2}{|c|}{$1.33 \pm 0.85$} & 0.001 \\
\hline \multicolumn{6}{|l|}{ Puntaje de MYOACT para actividad de la enfermedad } \\
\hline Total & \multicolumn{2}{|c|}{$6.5 \pm 2.25$} & \multicolumn{2}{|c|}{0} & 0.001 \\
\hline Actividad muscular & \multicolumn{2}{|c|}{$6.3 \pm 1.85$} & \multicolumn{2}{|c|}{0} & 0.001 \\
\hline Actividad cutánea & \multicolumn{2}{|c|}{$4.3 \pm 1.55$} & \multicolumn{2}{|c|}{0} & 0.001 \\
\hline
\end{tabular}

simultáneamente y por duplicado, desconociendo el estado clínico y la identidad del paciente. El valor definitivo de los niveles para cada caso se obtuvo al calcular la media de los dos valores obtenidos.

Después de la toma de la muestra, en los pacientes con enfermedad activa se inició tratamiento con $1 \mathrm{mg} / \mathrm{kg} / \mathrm{día}$ de prednisona por cuatro semanas con nueva toma de muestra de sangre.

El análisis estadístico se realizó mediante prueba exacta de Fisher para la comparación de variables categóricas y la prueba de rangos de Mann-Whitney para la comparación de variables numéricas. Los coeficientes de correlación se establecieron mediante la correlación de Spearman. Para comparar los promedios de las variables cuantitativas se utilizó la t de Student (entre grupos) y la t pareada (antes y después). Los datos fueron analizados con SPSS versión 22.0. Se consideró que una $p<0.05$ era estadísticamente significativa.

\section{Resultados}

Las características clínicas y bioquímicas de los pacientes se presentan en la Tabla 1. La media global de duración de la enfermedad fue de ocho meses (intervalo de dos a 17 meses). Al ser incluidos en el estudio, 28 pacientes presentaban enfermedad activa (grupo 1) y 17 enfermedad inactiva (grupo 2).

Los valores de las concentraciones séricas de C5b-9, así como de los niveles de creatinfosfoquinasa se presentan en la Tabla 1. Se observan diferencias significativas en los niveles de C5b-9 entre los casos con la enfermedad activa en comparación con aquellos con enfermedad inactiva $(p=0.008)$ y con los controles $(p=0.001)$ (Figura 1). Los niveles de creatinfosfoquinasa también mostraron diferencias significativas entre los tres grupos.

Los coeficientes de correlaciones del C5b-9 sérico con la severidad de la enfermedad dieron una correlación positiva, no así con los niveles de creatinfosfoquinasa; de la misma manera, este último parámetro mostró una baja correlación con la severidad clínica (Tabla 2).

Veintiocho pacientes con diagnóstico reciente de dermatomiositis se trataron con $1 \mathrm{mg} / \mathrm{kg} / \mathrm{día}$ de prednisona. Los niveles de C5b9 sérico al inicio del estudio (67.45 \pm 18.35$)$ fueron significativamente mayores a los medidos al término del tratamiento $(17.33 \pm 8.43$, $p=0.001$ ) (Figura 2). Los niveles de creatinfosfoquinasa no mostraron una reducción significativa al mes de tratamiento, aunque los parámetros clínicos de actividad (MYOACT) tuvieron una reducción significativa después del tratamiento con esteroides sistémicos (Tabla 3).

\section{Discusión}

Los niveles séricos de C5b-9 en los pacientes con dermatomiositis estuvieron más elevados que en los sujetos sanos, además, con este parámetro fue posible 
Tabla 2. Correlación entre los niveles séricos de C5b-9, creatinfosfoquinasa y marcadores clínicos en pacientes con dermatomiositis

\begin{tabular}{lccc}
\hline & Niveles de C5b-9 & Creatinfosfoquinasa & MYOACT total \\
\hline Niveles de C5b-9 & 1 & $0.122, p=0.063$ & $0.433, p=0.001$ \\
Creatinfosfoquinasa & $0.122, p=0.063$ & 1 & $0.231, p=0.05$ \\
MYOACT total & $0.433, p=0.001$ & $0.231, p=0.05$ & 1 \\
\hline
\end{tabular}

Las correlaciones se realizaron con la prueba de Pearson; significación estadística con $p<0.05$. MYOACT = marcadores clínicos

Tabla 3. Concentración sérica de C5b-9, creatinfosfoquinasa y MYOACT, antes y después de un mes de tratamiento con esteroides sistémicos en pacientes con dermatomiositis

\begin{tabular}{lccc}
\hline Variable & Antes del tratamiento & Un mes después del tratamiento & $\mathbf{p}$ \\
\hline Niveles séricos de C5b-9 $(\mathrm{ng} / \mathrm{mL})$ & $67.45 \pm 18.35$ & $17.33 \pm 8.43$ & 0.001 \\
Creatinfosfoquinasa $(\mathrm{mg} / \mathrm{dL})$ & $1675 \pm 1375$ & $1427 \pm 1239$ & 0.049 \\
MYOACT total & $6.5 \pm 2.25$ & $1.7 \pm 1.3$ & 0.001 \\
\hline
\end{tabular}

MYOACT $=$ Myositis Disease Activity Assessment

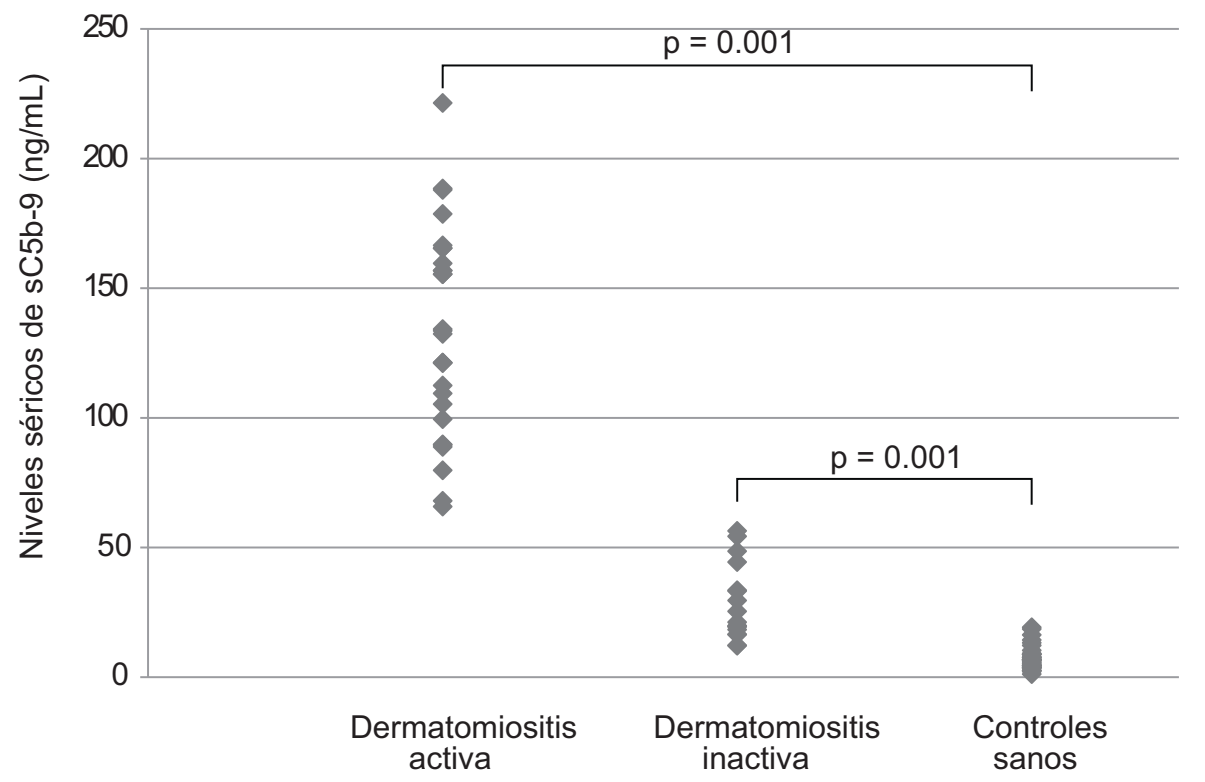

Figura 1. Niveles séricos de sC5b-9 en pacientes con dermatomiositis con enfermedad activa e inactiva y en sujetos sanos.

determinar los casos activos e inactivos de dermatomiositis. Otro aspecto importante es que el C5b-9 sérico puede ser un parámetro fiable de respuesta terapéutica, aún más preciso que la medición de enzimas musculares, particularmente creatinfosfoquinasa.

Los niveles séricos elevados de C5b-9 en pacientes con dermatomiositis confirman la activación del complemento en la patogenia de la enfermedad y tienden a estar más elevados en los pacientes con enfermedad activa que inactiva y en los controles sanos. En un trabajo previo de Campo et al. ${ }^{11}$ se observaron que los niveles séricos de C5b-9 correlacionaron con la severidad de la enfermedad, si bien los resultados no permitieron evaluar la respuesta al tratamiento, aun cuando es posible distinguir pacientes con enfermedad activa e inactiva. En nuestro estudio, además de observar buena correlación entre la actividad clínica y bioquímica de la enfermedad y el marcador, así como la capacidad de este para distinguir entre enfermedad activa e inactiva, fue de utilidad para evaluar la respuesta al tratamiento, con lo que podría ser de utilidad para determinar el pronóstico.

El C5b-9 es un mediador inflamatorio relacionado con la fisiopatología de muchas enfermedades. Las evidencias demuestran que el C5b-9 es un indicador de severidad clínica de las enfermedades con las que se ha relacionado, incluyendo lupus eritematoso sistémico y dermatomiositis, por lo que el bloqueo de este mediador 


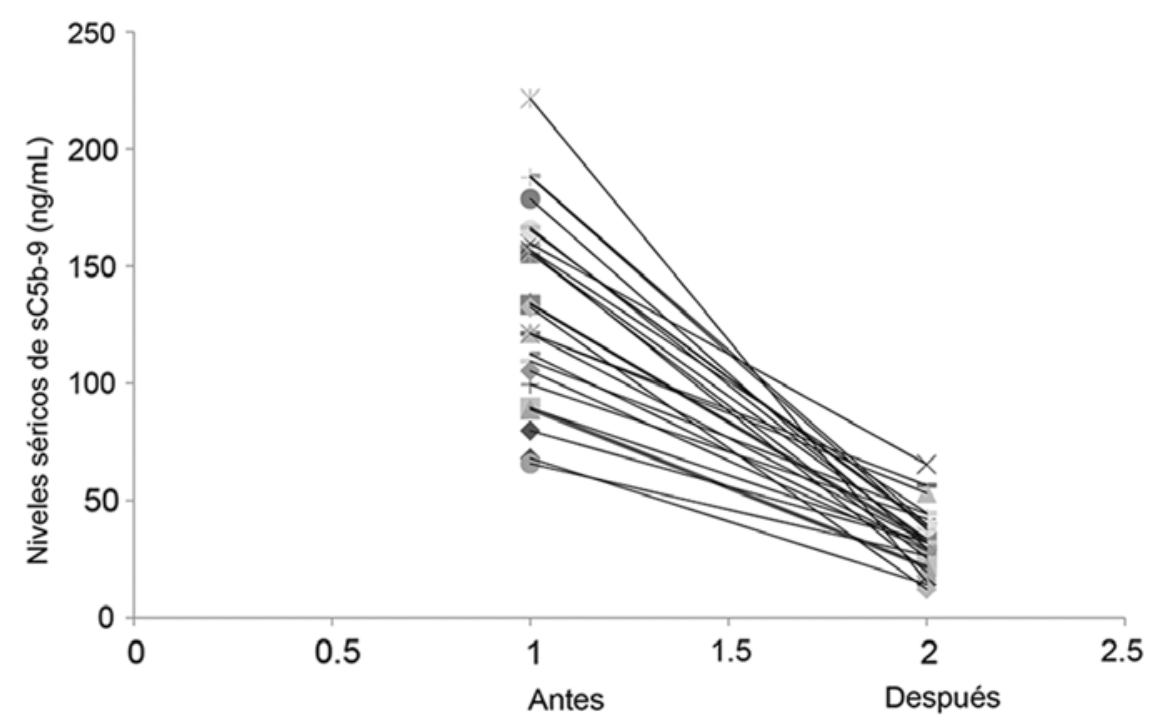

Figura 2. Niveles séricos de sC5b-9 después de un mes de tratamiento con esteroides sistémicos.

inflamatorio en muchos modelos de enfermedad inflamatoria contribuye a reducir la severidad de la misma. ${ }^{15}$

En nuestro estudio observamos correlación positiva entre los niveles séricos de C5b-9 y los signos de actividad de la enfermedad y, similar a lo reportado por Campo et al., ${ }^{11}$ este parámetro de activación del complemento no mostró correlación con los marcadores de actividad tradicionales, específicamente creatinfosfoquinasa.

En la dermatomiositis, las enzimas musculares son liberadas como consecuencia de la lesión de la fibra muscular, por lo que la correlación temporal entre su incremento y el desarrollo de debilidad muscular es importante. En los niveles de C5b-9 sérico, la elevación puede preceder a la actividad muscular y posiblemente a la actividad cutánea y sus niveles se mantienen durante la actividad de la enfermedad y correlacionan con la severidad.

El objetivo antigénico primario en la dermatomiositis es el endotelio de los capilares del endomisio. Estos anticuerpos producen la activación de C3, que a su vez provoca la formación de fragmentos C3b, C4b y de C5b-9 detectables en el curso de la enfermedad, tanto en el suero como en los capilares, incluso se observan en el tejido muscular antes que los cambios inflamatorios o estructurales. Estos depósitos son importantes para inducir la destrucción de las fibras musculares..$^{16}$

Otro mecanismo para explicar la relación entre C5b-9 y el desarrollo de la dermatomiositis se basa en la activación del complejo de ataque a la membrana que provoca un exceso de $\mathrm{Ca}^{2+}$, que da lugar a una sobrecarga mitocondrial, la pérdida del potencial transmembrana mitocondrial, dando como resultado una crisis energética dentro de las células, mientras que las bombas de iones tratan de restablecer la homeostasis del $\mathrm{Ca}^{2+}$; la liberación del citocromo $\mathrm{C}$ en el citoplasma y la activación de la caspasa desencadenan la apoptosis. Esto sugiere el papel crucial del C5b-9 en la activación del inflamasoma NALP3, con lo que amplifica la respuesta inflamatoria. ${ }^{17,18} \mathrm{El}$ inflamasoma NLRP3 desempeña un papel clave en el desarrollo de la dermatomiositis, ${ }^{19}$ lo que demuestra que la activación de C5b-9 puede preceder al desarrollo de las manifestaciones clínicas, bioquímicas e inmunológicas de la enfermedad.

Una limitante de nuestro estudio es que no fue posible comparar los niveles séricos de C5b-9 con los depósitos del mismo en piel y músculo, por lo que nuestro estudio podría servir de base para otros que evalúen si existe tal correlación. Asumimos que así es, ya que en estudios previos se observó que la biopsia del músculo de pacientes con dermatomiositis reveló el depósito del C5b-9 en las células endoteliales. ${ }^{20}$ Además, en biopsias de piel lesionada de pacientes con la enfermedad se observaron depósitos de C5b-9, no así en la piel no afectada, lo que sugiere que también estaría involucrado en la patogenia de las lesiones cutáneas. ${ }^{21}$

Por otra parte, dado que este estudio incluyó un solo centro, los hallazgos requieren confirmación en otros hospitales.

Los tratamientos actuales contra el complemento, especialmente el eculizumab, dirigidos específicamente a C5b-9 o a vías alternas se encuentran en desarrollo. ${ }^{15}$ 
En conclusión, la cuantificación de los niveles de los productos de activación del sistema del complemento es una herramienta para el control clínico de los pacientes con dermatomiositis, ya que permite distinguir entre enfermedad activa e inactiva y al correlacionar adecuadamente con la respuesta al tratamiento.

Por otro lado, es necesario determinar si los niveles séricos de C5b-9 podrían servir para distinguir otros tipos de miositis.

\section{Bibliografía}

1. Findlay AR, Goyal NA, Mozaffar T. An overview of polymyositis and dermatomyositis. Muscle Nerve 2015;51:638-656.

2. Luo YB, Mastaglia FL. Dermatomyositis, polymyositis and immune-mediatednecrotising myopathies. Biochim Biophys Acta. 2015;1852:622-632.

3. laccarino L, Ghirardello A, Bettio S, Zen M, Gatto M, Punzi L, et al. The clinical features, diagnosis and classification of dermatomyositis. J Autoimmun. 2014;48-49:122-127.

4. Lahoria R, Selcen D, Engel AG. Microvascular alterations and the role of complement in dermatomyositis. Brain. 2016;139(Pt 7):1891-1903.

5. Kissel JT, Halterman RK, Rammohan KW, Mendell JR. The relationship of complement-mediated microvasculopathy to the histologic features and clinical duration of disease in dermatomyositis. Arch Neurol. 1991;48:26-30.

6. Serna M, Giles JL, Morgan BP, Bubeck D. Structural basis of complementmembrane attack complex formation. Nat Commun. 2016;7:10587.

7. Dudkina NV, Spicer BA, Reboul CF, Conroy PJ, Lukoyanova N, Elmlund $\mathrm{H}$, et al. Structure of the poly-C9 component of the complement membrane attack complex. Nat Commun. 2016;7:10588.

8. Bayly-Jones C, Bubeck D, Dunstone MA. The mystery behind membrane insertion: a review of the complement membrane attack complex. Philos Trans R Soc Lond B Biol Sci. 2017;372:20160221.
9. Braczynski AK, Harter PN, Zeiner PS, Drott U, Tews DS, Preusse C, et al. C5b-9 deposits on endomysial capillaries in non-dermatomyositis cases. Neuromuscul Disord. 2016;26:283-291.

10. Sakuta R, Murakami N, Jin Y, Nagai T, Nonaka I, Nishino I. Diagnostic significance of membrane attack complex and vitronectin in childhood dermatomyositis. J Child Neurol. 2005;20:597-602.

11. Campo A, Hausmann G, Martí RM, Estrach T, Grau JM, Porcel JM, et al. Complement activation products (C3a and $\mathrm{C} 5 \mathrm{~b}-9)$ as markers of activity ofdermatomyositis. Comparison with usual biochemical parameters. Actas Dermosifiliogr. 2007;98:403-414.

12. Bohan A, Peter JB. Polymyositis and dermatomyositis (second of two parts). N Engl J Med. 1975;292:403-407.

13. Bohan A, Peter JB. Polymyositis and dermatomyositis (first of two parts). N Engl J Med 1975;292(7):344-7.

14. Isenberg DA, Allen E, Farewell V, Ehrenstein MR, Hanna MG, Lundberg IE, et al. International consensus outcome measures for patients with idiopathic inflammatory myopathies. Development and initial validation of myositis activity and damage indices in patients with adult onset disease. Rheumatology (Oxford). 2004;43:49-54.

15. Morgan BP. The membrane attack complex as an inflammatory trigger. Immunobiology. 2016;221:747-751.

16. Ballanti E, Perricone C, Greco E, Ballanti M, Di-Muzio G, Chimenti MS, et al. Complement and autoimmunity. Immunol Res. 2013;56:477-491.

17. Triantafilou K, Hughes TR, Triantafilou M, Morgan BP. The complement membrane attack complex triggers intracellular $\mathrm{Ca} 2+$ fluxes leading to NLRP3 inflammasome activation. J Cell Sci. 2013;126(Pt 13):2903-2913.

18. Zhao T, Gao J, Van J, To E, Wang A, Cao S, et al. Age-related increases in amyloid beta and membrane attack complex: evidence of inflammasome activation in the rodent eye. J Neuroinflammation. 2015;12:121.

19. Yin $X$, Han GC, Jiang $X W$, Shi $Q, P u C Q$. Increased expression of the NOD-like receptor family, pyrin domain containing 3 inflammasome in dermatomyositis and polymyositis is a potential contributor to their pathogenesis. Chin Med J (Engl). 2016;129:1047-1052.

20. Kissel JT, Mendell JR, Rammohan KW. Microvascular deposition of complement membrane attack complex in dermatomyositis. $\mathrm{N}$ Engl $\mathrm{J}$ Med. 1986;314:329-334.

21. Máscaro JM, Hausmann G, Herrero C, Grau JM, Cid MC, Palou J, et al. Membrane attack complex deposits in cutaneous lesions of dermatomyositis. Arch Dermatol. 1995;131:1386-1392. 